\title{
In vitro anti-inflammatory and anti-coagulant effects of antibiotics towards Platelet Activating Factor and thrombin
}

\author{
Alexandros B Tsoupras ${ }^{1 *}$, Maria Chini², Nickolaos Tsogas², Athina Lioni², George Tsekes², \\ Constantinos A Demopoulos ${ }^{1}$ and Marios C Lazanas $^{2}$
}

\begin{abstract}
Background: Sepsis is characterized as a systemic inflammatory response that results from the inability of the immune system to limit bacterial spread during an ongoing infection. In this condition the significant mediator of inflammation Platelet Activating Factor (PAF) and the coagulant factor thrombin are implicated. In animal models, treatment with PAF-antagonists or co-administration of antibiotics with recombinant-PAF-Acetylhydrolase (rPAF-AH) have exhibited promising results. In order to examine the putative anti-inflammatory and/or antithrombotic interactions between antibiotic treatment used in sepsis with PAF and/or thrombin, we studied the in vitro effects of these compounds towards PAF or/and thrombin related activities and towards PAF basic metabolic enzymes.
\end{abstract}

Methods: We assessed the inhibitory effect of these drugs against PAF or thrombin induced aggregation on washed rabbit platelets (WRPs) or rabbit Platelet Reach Plasma (rPRP) by evaluating their $I_{50}$ values. We also studied their effect on Cholinephosphotransferase of PAF (PAF-CPT)/Lyso-PAF-Acetyltransferase (Lyso-PAF-AT) of rabbit leukocytes (RLs), as well as on rabbit plasma-PAF-AH, the key enzymes of both de novo/remodelling PAF biosynthesis and PAF degradation, respectively.

Results: Several antibiotics inhibited PAF-induced platelet aggregation of both WRPs and rPRP in a concentrationdepended manner, with clarithromycin, azithromycin and amikacin exhibiting the higher inhibitory effect, while when combined they synergistically inhibited PAF. Higher concentrations of all antibiotics tested were needed in order to inhibit PAF induced aggregation of rPRP, but also to inhibit thrombin induced aggregation of WRPs. Concentrations of these drugs similar to their $\mathrm{IC}_{50}$ values against PAF activity in WRPs, inhibited also in vitro PAFCPT and Lyso-PAF-AT activities of rabbit leukocytes, while only clarithromycin and azithromycin increased rabbit plasma-PAF-AH activity.

Conclusions: These newly found properties of antibiotics used in sepsis suggest that apart from their general actions, these drugs may present additional beneficial anti-inflammatory and anti-coagulant effects against the onset and establishment of sepsis by inhibiting the PAF/PAF-receptor and/or the thrombin/protease-activatedreceptor-1 systems, and/or by reducing PAF-levels through both PAF-biosynthesis inhibition and PAF-catabolism induction. These promising in vitro results need to be further studied and confirmed by in vivo tests, in order to optimize the efficacy of antibiotic treatment in sepsis.

Keywords: Antibiotics, Lyso-PAF-AT, PAF, PAF-CPT, PAF-inhibitors, plasma-PAF-AH, Sepsis

\footnotetext{
* Correspondence: atsoupras@yahoo.gr

'Faculty of Chemistry, National \& Kapodistrian University of Athens,

Panepistimioupolis of Zografou, Athens, 15771, Greece

Full list of author information is available at the end of the article
} 


\section{Background}

Platelet Activating Factor (PAF) is a phospholipid signalling molecule of inflammation and a significant mediator of the immune system [1,2]. PAF transmits outside-in signals to intracellular transduction systems in a variety of cell types, including key cells of the innate immune and haemostatic systems: neutrophils, monocytes, and platelets [2]. Binding of PAF on specific membrane receptors coupled with G-proteins (PAF-receptor, PAFR) induces several intracellular signaling pathways that leads to auto/endo/para/juxta-crine cellular activation [3].

PAF can be synthesized by two different and distinct enzymatic routes, namely the remodeling and the de novo pathway [4-6]. The remodeling pathway involves a structural modification of 1-O-ether-linked membrane phospholipids where the action of cytoplasmic phospholipase A2 yields lyso-PAF which is then acetylated by a lyso-PAF:acetyl-CoA acetyltransferase (Lyso-PAF AT, EC 2.3.1.67) leading to the formation of PAF. In the de novo pathway, PAF-production occurs from simple molecules such as alkylglycerophosphate (AGP) in several steps. A central step is the conversion of 1-O-alkyl-2-acetyl-glycerol to PAF by a specific dithiothreitol-insensitive CDPcholine: 1-alkyl-2-acetyl-sn-glycerol cholinephosphotransferase (PAF-CPT, EC 2.7.8.16). Concerning PAF catabolism the most important enzyme involved is a PAFspecific acetylhydrolase (PAF-AH, EC 3.1.1.47), which cleaves the short acyl chain at the sn-2 position and forms lyso-PAF, which is biologically inactive [7].

Increased levels of PAF are implicated in several diseases, mainly inflammatory but also non-inflammatory ones [1-3], such as cardiovascular, renal and periodontal diseases [8-11], allergy [12], diabetes [13], cancer [14], AIDS [15] and Sepsis [16-23].

A great variety of molecules have been found to exhibit an inhibitory effect on PAF-induced biological activities, acting either through their direct antagonistic/ competitive effect to PAF by binding on PAFR, or through other indirect mechanisms [24], that have not been fully clarified but seems to correlate with changes in the membrane microenvironment of PAF-receptor. Blockage of PAFR by such molecules represents a new therapeutic approach against several of the above mentioned diseases including Sepsis [16-23]. In addition, various PAF-inhibitors exhibit also the ability to in vitro and in vivo inhibit PAF-CPT, Lyso-PAF-AT and/or to induce PAF-AH activities [[15,25] unpublished data by AB Tsoupras).

Pharmacological data obtained with PAF antagonists, indicate a significant role for PAF in sepsis, septic shock and in the priming process [16-23]. Sepsis is a systemic inflammatory response that results from the inability of the immune system to limit bacterial spread during an ongoing infection. The effect of PAF antagonists in different models of sepsis and shock states indicates a role for PAF in endotoxin associated lethality, activation of inflammatory blood cells with release of mediators, cardiovascular failure and increased vascular permeability, as well as in the development of shock organs and organ failure.

The precise role of PAF as mediator of the diffuse inflammatory state characteristic of sepsis remains to be determined, but, in animal models, beneficial effects have been observed as a result of treatment with various antagonists of PAF [16-23]. Strategies to block inflammatory mediators such as PAF, often with complicated outcomes, are currently being investigated as new adjuvant therapies for sepsis. To date, however, it has been impossible to duplicate these encouraging results from animal models in the clinical setting.

On the other hand, administration of recombinant PAF-AH (rPAF-AH), protects mice from inflammatory injury and death after administration of lipopolysaccharide (LPS) or cecal ligation and puncture (CLP) [26]. Coadministration of antibiotics together with rPAF-AH was more effective than single treatment with either of these agents [26]. The beneficial effects of this combined treatment suggest a potential role of antibiotics against PAF implication in sepsis.

In order to examine the possible interactions between PAF and antibiotic treatment against sepsis we studied their potential effect on PAF-metabolism and/or their putative anti-PAF activity.

For this reason in the present study we examined for the first time the in vitro anti-inflammatory and antithrombotic ability of a broad-spectrum of antibiotics and several of their combinations/regimens used in treatment against sepsis, based on their effect towards PAF-induced or thrombin induced platelet aggregation of Washed Rabbit Platelets (WRPs) and rabbit Platelet Reach Plasma (rPRP). In addition we examined their ability to affect PAF-metabolism by decreasing PAFactivity, through their in vitro effect on PAF basic metabolic enzymes, PAF-CPT and lyso PAF-AT of rabbit leukocytes as well as rabbit plasma PAF-AH.

\section{Materials and methods \\ Materials and instruments}

Centrifugations were performed in an Heraeus Labofug $400 \mathrm{R}$ and a Sorvall RC-5B refrigerated super speed centrifuge Homogenizations were conducted in a supersonic sonicator (Sonics \& Materials, Newtown, CT, USA). The liquid scintillation counter used was a 1209 Rackbeta (Pharmacia, Wallac, Finland). PAF-induced platelet aggregation studies were performed in a model 400 VS 
aggregometer of Chrono-Log (Havertown, PA, USA) coupled to a Chrono-Log recorder at $37^{\circ} \mathrm{C}$ with constant stirring at $1200 \mathrm{rpm}$.

BSA (bovine serum albumin), PAF (1-O-hexadecyl-2acetyl-sn-glycero-3-phosphocholine), thrombin, trichloroacetic acid (TCA), CDP-choline, lyso-PAF, acetyl-CoA, dithiothreitol (DTT), EDTA, $\mathrm{MgCL}_{2}$, Tris and analytical reagents and solvents were purchased from Sigma (St. Louis, MO, USA). 1-O-hexadecyl-2- $\left[{ }^{3} \mathrm{H}\right]$ acetyl-sn-glycerol-3-phosphocholine $\left(\left[{ }^{3} \mathrm{H}\right] \mathrm{PAF}\right)$ with a specific activity of $10 \mathrm{Ci} / \mathrm{mmol}$ was obtained from New England Nuclear (Dupont, Boston, MA, USA). 1-O-alkyl-2-snacetyl-glycerol (AAG) was purchased from BIOMOL International LP (Palatine House, Matford Court, Exeter, UK). 2,5-Diphenyloxazole (PPO) and 1,4-bis(5-phenyl-2oxazolyl) benzene (POPOP) were purchased from $\mathrm{BDH}$ Chemicals (Dorset, England). Scintillation liquid cocktail (dioxane base) was prepared by diluting $7 \mathrm{~g}$ PPO, $0.3 \mathrm{~g}$ POPOP and $100 \mathrm{~g}$ Napthalene in $200 \mathrm{~mL} \mathrm{H}_{2} \mathrm{O}$ and then transferred to $1 \mathrm{~L}$ of dioxane. Liquid chromatography grade solvents and silica G for TLC were purchased from Merck KGaA (Darmstadt, Germany).

The antibiotics that were tested were provided by our hospital pharmacy and were dissolved in $2.5 \mathrm{mg}$ bovine serum albumin $(\mathrm{BSA}) / \mathrm{mL}$ saline $[1,15]$. In order to test several combinations of antibiotic regimens, several mixtures of these drugs were also prepared using the above solutions of each drug. The ratios of the concentrations $(\mu \mathrm{g} / \mu \mathrm{L})$ of the active components that were used in each mixture are shown in Table two.

\section{Biological assays on Washed Rabbit Platelets (WRPs) and rabbit Platelet Reach Plasma (rPRP)}

We assessed the in vitro inhibitory effect of these drugs and their combinations in anti-septic treatment regimens against PAF-induced or thrombin induced aggregation on WRPs and rPRP by evaluating the concentration $(\mu \mathrm{g} / \mathrm{mL})$ of the bioactive compound $(\mathrm{s})$ in each case in the aggregometer cuvette that inhibited $50 \%$ PAF-induced or thrombin induced aggregation $\left(\mathrm{IC}_{50}\right)$ of WRPs or rPRP, as previously described $[1,15,27,28]$. Briefly, PAF and the examined drugs were dissolved in $2.5 \mathrm{mg} \mathrm{BSA} / \mathrm{ml}$ saline. The drugs were tested for their ability to inhibit PAF-induced aggregation of WRPs or rPRP and thrombin induced aggregation of WRPs and/or to induce WRPs aggregation in a Chrono-Log aggregometer. Various concentrations of the examined samples were added into the aggregometer cuvette 1 min prior to the addition of PAF or thrombin. The platelet aggregation induced by PAF (4.4 $\times 10^{-11} \mathrm{M}$ and $2.24 \times 10^{-7} \mathrm{M}$, final concentration in the aggregometer cuvette in the cases of WRPs and rPRP respectively) or thrombin $(0.01 \mathrm{mU}$ in the aggregometer cuvette in the case of WRPs) was measured as PAF- induced or thrombin induced aggregation in WRPs or rPRP before (considered as $0 \%$ inhibition) and after the addition of various concentrations of the examined sample $[15,27,28]$. A linear plot of inhibition percentage (ranging from $20 \%$ to $80 \%$ ) versus the concentration of the sample was established for each antibiotic and in each case. From this curve, the concentration of the sample that inhibited $50 \%$ of the PAF or thrombin induced aggregation $\left(\mathrm{IC}_{50}\right)$ was calculated. The aggregatory activity of the sample was expressed as micrograms of the bioactive compound(s) of the drugs dissolved in $2.5 \mathrm{mg} \mathrm{BSA} / \mathrm{ml}$ saline, which is able to induce $50 \%$ of the maximum reversible aggregation of the respective sample, defined as $\mathrm{EC}_{50}$ value. In addition, desensitization tests were carried out as previously described $[15,27]$. Briefly, in desensitization and cross-desensitization experiments, platelets were activated by the addition of PAF or drugs to the platelet suspension at a concentration that caused reversible aggregation. Second stimulation with the tested bioactive compound(s) or PAF respectively, was performed immediately after complete disaggregation.

\section{Isolation of plasma and leukocytes from rabbit blood}

The isolation of plasma and leukocytes from rabbit blood was performed as previously described [15] with some modifications. Briefly: $9 \mathrm{~mL}$ of blood were obtained from each rabbit in $1 \mathrm{~mL}$ of an anticoagulant solution of sodium citrate/citrate acid.

The sample was centrifuged at $630 \mathrm{~g}$ for $10 \mathrm{~min}$ at $25^{\circ}$ C (1st centrifugation). The supernatant (plasma reach in platelets) was centrifuged at $1400 \mathrm{~g}$ for $20 \mathrm{~min}$ at $25^{\circ} \mathrm{C}$ (2nd centrifugation).

The supernatant of the 2 nd centrifugation (plasma) was aliquoted and stored at $-80^{\circ} \mathrm{C}$ until the time of the plasma PAF-AH assay analysis.

From the pellet of the 1st centrifugation (leukocytes and erythrocytes) the isolation of the leukocytes from the contaminating erythrocytes was achieved by erythrocyte sedimentation. Saline was added in order the sample reached its initial volume of $10 \mathrm{~mL}$. The sample was separated in half and $1.7 \mathrm{~mL}$ of dextran solution (3\% dextran in $\mathrm{NaCl} 0.15 \mathrm{M}$ ) was added in each half and the mixtures were kept for $1 \mathrm{~h}$ at room temperature. The leukocyte-rich supernatants were then centrifuged at $500 \mathrm{~g}$ for $10 \mathrm{~min}$ at room temperature (4th centrifugation). Contaminating erythrocytes of the sediment were lysed with the addition of a lysis solution consisting of $155 \mathrm{mM} \mathrm{NH}_{4} \mathrm{Cl}, 10 \mathrm{mM} \mathrm{KHCO}_{3}$, and $0.1 \mathrm{mM}$ EDTA and then removed with a centrifugation at $300 \mathrm{~g}$ for 10 min at room temperature (5th centrifugation).

The pelleted cells of the 5 th centrifugation (isolated leukocytes) were resuspended in $1 \mathrm{ml}$ of a buffer containing $50 \mathrm{mM}$ Tris- $\mathrm{HCl}(\mathrm{pH} 7.4)$ and sonicated on ice 
$(4 \times 15 \mathrm{~s})$. Then they were centrifuged at $500 \mathrm{~g}$ for 10 min at $4^{\circ} \mathrm{C}$ (6th centrifugation) in order to remove whole cells, nucleuses and debris in the pellet and the supernatants (homogenates) after protein determination were aliquoted and stored at $-80^{\circ} \mathrm{C}$ until the time of the PAF-CPT and Lyso-PAF-AT assays analysis.

\section{DTT-insensitive PAF-Cholinephosphotransferase (PAF-CPT)} activity assays

Assay was performed on the homogenates of rabbit leukocytes as previously described $[15,25]$. Briefly, the reaction was carried out at $37^{\circ} \mathrm{C}$ for $20 \mathrm{~min}$ in a final volume of $200 \mu \mathrm{L}$ containing $0.05-2.5 \mathrm{mg} / \mathrm{mL}$ protein, $100 \mathrm{mM}$ Tris- $\mathrm{HCl}$ (pH 8.0), $15 \mathrm{mM}$ dithiothreitol (DTT), $0.5 \mathrm{mM}$ EDTA, $20 \mathrm{mM} \mathrm{MgCl}_{2}, 1 \mathrm{mg} / \mathrm{mL} \mathrm{BSA}$, $100 \mu \mathrm{M}$ CDP-Choline and $100 \mu \mathrm{M}$ 1-O-alkyl-2-snacetyl-glycerol (AAG). The reaction was stopped by adding $0,5 \mathrm{ml}$ of cold methanol ( $2 \%$ acetic acid). The extraction, purification and determination of PAF were performed as previously described [25]. Briefly, 0,25 ml of cold chloroform was added in order to firstly reach the proportion of $1 / 2 / 0.8 \mathrm{CHCl}_{3}: \mathrm{MeOH}: \mathrm{H}_{2} \mathrm{O}$, and after potent vortex another $0,25 \mathrm{ml}$ of cold chloroform and $0,25 \mathrm{ml}$ of water were added in order to finally reach the proportion of $1 / 1 / 0.9 \mathrm{CHCl}_{3}: \mathrm{MeOH}: \mathrm{H}_{2} \mathrm{O}$ from where produced PAF was extracted in the chloroform phase by the acid Bligh-Dyer method [29]. The extracted PAF was further separated by thin-layer chromatography (TLC) on Silica Gel G coated plates with an elution system consisting of chloroform:methanol:acetic acid: water (100:57:16:8, v/v/v/v). The band corresponding to PAF (between lyso-phosphatidylcholine and phosphatidylcholine) was identified by co-chromatographing lipid standards which were visualized by exposure of the plates to iodine vapors. PAF fractions were scrapped off, extracted by the Bligh-Dyer method [29] and the amount of PAF was determined by the washed rabbit platelet aggregation assay [1]. All assays were performed in duplicate. Enzymatic activities were expressed as specific activity in $\mathrm{nmol} / \mathrm{min} / \mathrm{mg}$ of total protein.

The effect of drugs on PAF-CPT activity was evaluated in homogenates of rabbit leukocytes. The in vitro enzymatic assay of PAF-CPT was performed in the presence of several concentrations of each drug in the assay reaction mixture as previously described [15].

\section{Lyso-PAF-AT activity assays}

Assay was performed on the homogenates of leukocytes as previously described [15]. Briefly, the reaction was carried out at $37^{\circ} \mathrm{C}$ for $30 \mathrm{~min}$ in a final volume of 200 $\mu \mathrm{L}$ containing $0.05-2.5 \mathrm{mg} / \mathrm{mL}$ protein, $50 \mathrm{mM}$ Tris$\mathrm{HCl}$ (pH 7,4), $0.25 \mathrm{mg} / \mathrm{mL}$ BSA, $20 \mu \mathrm{M}$ Lyso-PAF and $200 \mu \mathrm{M}$ acetyl-CoA. The reaction was stopped by adding $2 \%$ acetic acid methanol and the extraction, purification and determination of PAF was carried out as mentioned above in the PAF-CPT-assay [25]. All assays were performed in duplicate. Enzymatic activities were expressed as specific activity in $\mathrm{nmol} / \mathrm{min} / \mathrm{mg}$ of total protein.

The effect of drugs on Lyso-PAF-AT activity was also evaluated in homogenates of rabbit leukocytes. The in vitro enzymatic assay of Lyso-PAF-AT was performed in the presence of several concentrations of each drug in the assay reaction mixture as previously described [15].

\section{Plasma PAF-AH activity assays}

Plasma-PAF-AH activity was determined by the trichloroacetic acid precipitation method using $\left[{ }^{3} \mathrm{H}\right]-\mathrm{PAF}$ as a substrate as previously described [30]. Briefly, $2 \mu \mathrm{L}$ of plasma were incubated with $4 \mathrm{nmol}$ of $\left[{ }^{3} \mathrm{H}\right] \operatorname{PAF}(20 \mathrm{~Bq}$ per nmol) for $30 \mathrm{~min}$ at $37^{\circ} \mathrm{C}$ in a final volume of 200 $\mu \mathrm{L}$ of $50 \mathrm{mM}$ Tris/ $\mathrm{HCl}$ buffer $(\mathrm{pH} 7.4)$. The reaction was terminated by the addition of cold trichloroacetic acid (10\% final concentration). The samples were then placed in an ice bath for $30 \mathrm{~min}$ and subsequently centrifuged at $16000 \mathrm{~g}$ for $5 \mathrm{~min}$. The $\left[{ }^{3} \mathrm{H}\right]$-acetate released into the aqueous phase was measured on a liquid scintillation counter. All assays were performed in duplicate. The enzyme activity was expressed as nmol of PAF degraded per min per $\mathrm{mL}$ of plasma.

The effect of drugs on PAF-AH activity was evaluated in rabbit plasma. The in vitro enzymatic assay of plasma PAF-AH was performed in the presence of several concentrations of each drug in the assay reaction mixture as previously described [15].

\section{Analytical methods}

Protein concentrations, determined according to the method of Bradford [31], were based on BSA as the protein standard.

\section{Statistical analysis}

Normal distribution of variables was checked using Kolmogorov-Smirnov criterion before further analyses. Data are expressed as geometrical mean with $95 \%$ confidence limits along with median, minimum and maximum values for IC50 values and as mean values \pm SD for enzyme activities. Differences in PAF-metabolic enzymes activities in the presence and in the absence (control) of drugs were assessed by multiple comparisons with one way ANOVA using LSD post-hoc tests and were considered to be statistically significant when $\mathrm{p}<0.05$. Data were analyzed using a statistical software package, SPSS 18.0, and Microsoft Excel 2007 for Windows.

\section{Results}

Several antibiotics inhibited in vitro PAF induced aggregation of washed rabbit platelets in a concentrationdependant manner. Their $\mathrm{IC}_{50}$ values against PAF are expressed as micrograms $/ \mathrm{mL}(\mu \mathrm{g} / \mathrm{mL})$ of bioactive 
compound in the aggregometer cuvette that cause 50\% inhibition of PAF-induced washed rabbit platelet aggregation in a final concentration of $4.4 \times 10^{-11} \mathrm{M}$ (Table 1). The $\mathrm{IC}_{50}$ values ranged from 0.19 to 110.95 $\mu \mathrm{g} / \mathrm{mL}$, approximately. The most potent ones in the rank were clarithromycin, azithromycin, linezolid, amicacin and netilmycin. Other drugs studied such as meropenem and vancomycin, did not influence PAF activity in WRPs.

From all antibiotics tested, only vancomycin, induced aggregation in WRPs in a concentration much higher than its $\mathrm{IC}_{50}$ value (Table 1). Desensitization and cross desensitization experiments showed that vancomycin seemed to induce platelet aggregation through a different way than that of PAF pathway (Table 1).

All antibiotics were further tested for their potential inhibitory effect against the PAF-induced rabbit PRP aggregation. Their $\mathrm{IC}_{50}$ values in this case are also expressed as micrograms $/ \mathrm{mL}(\mu \mathrm{g} / \mathrm{mL})$ of bioactive compound in the aggregometer cuvette that cause $50 \%$ inhibition of PAF-induced aggregation of rPRP in a final concentration of $2.24 \times 10^{-7} \mathrm{M}$ (Table 2). These $\mathrm{IC}_{50}$ values ranged from 8.3 to $829.0 \mu \mathrm{g} / \mathrm{mL}$, approximately. In the case of rPRP the most potent antibiotics in the rank were amicacin, azithromycin, tygecycline and clarithromycin, while other drugs studied such as meropenem and linezolid, did not influence PAF activity in rPRP.

All antibiotics were also tested for their potential inhibitory effect towards the thrombin induced WRP's aggregation. Their $\mathrm{IC}_{50}$ values in this case are also expressed as micrograms $/ \mathrm{mL}(\mu \mathrm{g} / \mathrm{mL})$ of bioactive compound in the aggregometer cuvette that cause $50 \%$ inhibition of thrombin-induced aggregation of WRPs in a final concentration of $0.01 \mathrm{mU}$ (Table 3). These $\mathrm{IC}_{50}$ values ranged from 6.7 to $350.3 \mu \mathrm{g} / \mathrm{mL}$, approximately. In this case, the most potent antibiotics in the rank were netilmicin, azithromycin, amicacin and daptomycin, while again meropenem did not influence thrombin activity in WRPs.

Several combinations of these drugs were also tested against PAF-induced aggregation of WRPs. The most potent ones are presented in Table 4. Among the combinations of antibiotics of regimens against sepsis that were tested, piperacillin-tazobactam/netilmicin, piperacillin-tazobactam/amikacin, ceftazidime/amikacin, ceftazidime/netilmicin displayed the higher inhibitory effect against PAF activity in WRPs, respectively (Table 4).

In addition when these drugs were added, in concentrations similar to their $\mathrm{IC}_{50}$ values against PAF activity, in the enzymatic assays of both PAF-CPT and LysoPAF-AT of rabbit leukocytes they in vitro significantly inhibited both enzymes activities in a concentration depended manner $(\mathrm{p}<0.05$ in relevance to control assays). In Figures 1 and 2 are shown the amounts of each drug that induced approximately fifty to one hundred inhibitory effect against PAF-CPT and Lyso-PAFAT specific activities respectively (Figures 1,2 ). Moreover, the amount of clarithromycin needed in order to achieve this inhibition in both PAF-CPT and Lyso-PAF$\mathrm{AT}$ was found one order of magnitude lower than those of all the other antibiotics, with the exception of that of amikacin in the case of Lyso-PAF-AT inhibition, which in turn was also much lower than those of all the other antibiotics tested.

Table 1 In vitro inhibitory effect (expressed as IC $_{50}$ ) of the antibiotics tested against PAF-induced aggregation of WRPs and their ability to induce platelet aggregation

\begin{tabular}{|c|c|c|c|c|c|c|}
\hline \multirow[b]{2}{*}{$\begin{array}{l}\text { Bioactive } \\
\text { Compound }\end{array}$} & \multicolumn{5}{|c|}{$\mathrm{IC}_{50}{ }^{1}$ towards PAF in WRPs $(\mu \mathrm{g} / \mathrm{mL})$} & \multirow[b]{2}{*}{$\begin{array}{c}\text { Drug-induced WRPs aggregation } \\
\text { desensitization }\end{array}$} \\
\hline & Median & Min & Max & $\begin{array}{c}\text { Geometric } \\
\text { Mean }\end{array}$ & 95\% Confidence Interval & \\
\hline Clarithromycin & 0.18 & 0.14 & 0.28 & 0.19 & 0.08 thru 0.46 & $-/$ \\
\hline Azithromycin & 0.40 & 0.20 & 0.85 & 0.41 & 0.07 thru 2.46 & - \\
\hline Linezolid & 1.25 & 0.60 & 1.62 & 1.07 & 0.30 thru 3.84 & $-/-$ \\
\hline Amikacin & 2.73 & 1.50 & 4.55 & 2.65 & 0.67 thru 10.54 & $-/$ \\
\hline Netilmicin & 2.80 & 1.45 & 4.70 & 2.67 & 0.62 thru 11.56 & $-/-$ \\
\hline Daptomycin & 5.01 & 2.88 & 7.22 & 4.71 & 1.49 thru 14.85 & $-/$ \\
\hline $\begin{array}{l}\text { Piperacillin/ } \\
\text { Tazobactam }\end{array}$ & $\begin{array}{c}17.65 / \\
2.22\end{array}$ & $\begin{array}{c}12.18 / \\
1.54\end{array}$ & $\begin{array}{l}22.27 / \\
2.85\end{array}$ & $16.85 / 2.14$ & $\begin{array}{c}7.91 \text { thru } 35.90 / 0.99 \text { thru } \\
4.61\end{array}$ & $-/-$ \\
\hline Ceftazidime & 30.06 & 20.92 & 37.95 & 28.79 & 13.66 thru 60.68 & $-/-$ \\
\hline Tigecycline & 113.45 & 91.86 & 131.07 & 110.95 & 71.16 thru 173.0 & $-/-$ \\
\hline Vancomycin & ND & - & - & - & - & $+/-$ \\
\hline Meropenem & ND & - & - & - & - & $-/-$ \\
\hline
\end{tabular}

Experiments were conducted three times using different platelets preparations. ${ }^{1} \mathrm{I} \mathrm{C}_{50}$ values are expressed as $\mu \mathrm{g} / \mathrm{mL}$ of bioactive compound in the aggregometer cuvette, Final concentration of PAF in the aggregometer cuvette when tested in WRPs was $4.4 \times 10^{-11} \mathrm{M}$. WRPs: Washed Rabbit Platelets; ND: Not detected inhibition against PAF-induced platelet aggregation; -/-: Not detected platelet aggregation; +/-: Detected platelet aggregation/not detected platelet desensitization against PAF. 
Table 2 In vitro inhibitory effect (expressed as $I_{50}$ ) of the antibiotics tested against PAF-induced aggregation of rPRP

\begin{tabular}{|c|c|c|c|c|c|}
\hline \multirow[b]{2}{*}{$\begin{array}{l}\text { Bioactive } \\
\text { Compound }\end{array}$} & \multicolumn{5}{|c|}{$\mathrm{IC}_{50}{ }^{1}$ towards PAF in $\mathrm{rPRP}(\mu \mathrm{g} / \mathrm{mL})$} \\
\hline & Median & Min & Max & Geometric Mean & 95\% Confidence Interval \\
\hline Clarithromycin & 49.6 & 33.2 & 78.4 & 50.5 & 17.4 thru 147.1 \\
\hline Azithromycin & 23.3 & 11.9 & 29.6 & 20.2 & 6.2 thru 65.2 \\
\hline Linezolid & ND & - & - & - & - \\
\hline Amikacin & 9.6 & 5.4 & 11.2 & 8.3 & 3.2 thru 21.7 \\
\hline Netilmicin & 384.6 & 365.9 & 430.4 & 392.7 & 319.4 thru 482.9 \\
\hline Daptomycin & 384.5 & 375.8 & 465.8 & 406.8 & 303.5 thru 545.2 \\
\hline Piperacillin/Tazobactam & $837.1 / 86.4$ & $765.0 / 76.9$ & $889.6 / 102.3$ & $829.0 / 87.9$ & 686.5 thru $1001.0 / 61.6$ thru 125.6 \\
\hline Ceftazidime & 385.5 & 345.6 & 412.9 & 380.3 & 304.3 thru 475.3 \\
\hline Tigecycline & 26.0 & 20.8 & 27.3 & 24.6 & 17.1 thru 35.2 \\
\hline Vancomycin & 70.9 & 62.1 & 73.7 & 68.7 & 55.0 thru 86.0 \\
\hline Meropenem & ND & - & - & - & - \\
\hline
\end{tabular}

Experiments were conducted three times using different platelets preparations. ${ }^{1} \mathrm{I} \mathrm{C}_{50}$ values are expressed as $\mu \mathrm{g} / \mathrm{mL}$ of bioactive compound in the aggregometer cuvette. Final concentration of PAF in the aggregometer cuvette when tested when tested in rPRP was $2.24 \times 10^{-7} \mathrm{M}$. rPRP: rabbit Platelet Reach Plasma; ND: Not detected inhibition against PAF-induced platelet aggregation.

On the other hand, from the entire drug tested only clarithromycin and azithromycin induced an in vitro significant increase of rabbit plasma PAF-AH ( $<0.05$ in relevance to control assays), in concentrations of an order of magnitude higher than their $\mathrm{IC}_{50}$ values against PAF. In Figure 3 are shown the amounts of these two drugs that induced the significant increase of rabbit plasma PAF-AH enzyme activity (Figure 3). Moreover, the amount of clarithromycin needed in order to achieve this induction in plasma-PAF-AH was found one order of magnitude lower than that of azithromycin $(\mathrm{p}<0.05)$

\section{Discussion}

Sepsis is a systemic inflammatory response that results from the inability of the immune system to limit bacterial spread during an ongoing infection. The pathophysiology of sepsis is not completely understood. Bacteria are the main cause of sepsis. Activated receptors of the innate immune system lead to an exaggerated immune response including systemic inflammation. Immune cells including activated neutrophils and macrophages express and are controlled by a variety of cytokines, chemokines, complement factors and other mediators such as PAF and Thrombin [16-23,32]. The activation of toll-like receptors such as TLR4 usually leads to further amplification of inflammation through these mediators [32]. These receptors have been found to be directly activated by bacteria Lipopolysaccharide (LPS) and thus inducing PAF biosynthesis by the phosphorylation and subsequently activation of Lyso-PAF-AT enzyme activity [33].

Table 3 In vitro inhibitory effect (expressed as $I C_{50}$ ) of the antibiotics tested against thrombin induced aggregation of WRPs

\begin{tabular}{|c|c|c|c|c|c|}
\hline \multirow[b]{2}{*}{$\begin{array}{l}\text { Bioactive } \\
\text { Compound }\end{array}$} & \multicolumn{5}{|c|}{$\mathrm{IC}_{50}{ }^{1}$ towards Thrombin in WRPs $(\mu \mathrm{g} / \mathrm{mL})$} \\
\hline & Median & Min & Max & Geometric Mean & 95\% Confidence Interval \\
\hline Clarithromycin & 105.6 & 88.3 & 119.5 & 103.7 & 71.0 thru 151.3 \\
\hline Azithromycin & 13.6 & 11.9 & 14.5 & 13.3 & 10.3 thru 17.1 \\
\hline Linezolid & 98.0 & 93.0 & 110.1 & 100.1 & 80.8 thru 124.1 \\
\hline Amikacin & 22.0 & 18.7 & 27.3 & 22.4 & 14.0 thru 36.0 \\
\hline Netilmicin & 6.6 & 5.7 & 8.1 & 6.7 & 4.3 thru 10.4 \\
\hline Daptomycin & 42.7 & 33.7 & 45.9 & 40.4 & 27.1 thru 60.4 \\
\hline Piperacillin/Tazobactam & $142.3 / 17.8$ & $123.6 / 15.6$ & $170.1 / 20.8$ & $144.1 / 17.9$ & 96.8 thru $214.4 / 12.5$ thru 25.7 \\
\hline Ceftazidime & 99.2 & 82.8 & 115.3 & 98.2 & 65.0 thru 148.3 \\
\hline Tigecycline & 262.0 & 222.7 & 311.6 & 262.9 & 173.2 thru 399.1 \\
\hline Vancomycin & 354.0 & 312.7 & 388.5 & 350.3 & 267.3 thru 459.2 \\
\hline Meropenem & ND & - & - & - & - \\
\hline
\end{tabular}

Experiments were conducted three times using different platelets preparations. ${ }^{1} \mathrm{I} \mathrm{C}_{50}$ values are expressed as $\mu \mathrm{g} / \mathrm{mL}$ of bioactive compound in the aggregometer cuvette. Final concentration of thrombin in the aggregometer cuvette was $0.01 \mathrm{mU}$ in WRPs. WRPs: Washed Rabbit Platelets; ND: Not detected inhibition against thrombin-induced platelet aggregation. 
Table 4 In vitro inhibitory effect (expressed as $\mathrm{IC}_{50}$ ) of the most potent combinations of antibiotic anti-septic regimens against PAF-induced WRPs aggregation

\begin{tabular}{ccccccc}
\hline & \multicolumn{7}{c}{ IC $_{\mathbf{5 0}}{ }^{2}$ towards PAF in WRPs $(\boldsymbol{\mu g} / \mathbf{m L}$ ) } & & \\
Combinations of Bioactive Compounds & Ratio $^{1}$ & Median & Min & Max & Geometric Mean & 95\% Confidence Interval \\
\hline Piperacillin-Tazobactam/Netilmicin & $40-5 / 1$ & $5.1-0.6 / 0.1$ & $4.6-0.6 / 0.1$ & $5.3-0.7 / 0.1$ & $5.0-0.6 / 0.1$ & 4.2 thru 6.0-0.5 thru 0.8/0.1 thru 0.1 \\
Piperacillin-Tazobactam/Amikacin & $40-5 / 1.7$ & $5.1-0.6 / 0.2$ & $4.6-0.6 / 0.2$ & $5.4-0.7 / 0.2$ & $5.1-0.6 / 0.2$ & 4.2 thru 6.1-0.5 thru 0.8/0.2 thru 0.2 \\
Ceftazidime/Amikacin & $6 / 1$ & $10.0 / 1.7$ & $7.4 / 1.2$ & $13.1 / 2.6$ & $9.9 / 1.7$ & 4.9 thru 20.1/0.7 thru 4.6 \\
Ceftazidime/Netilmicin & $10 / 1$ & $10.6 / 1.1$ & $7.8 / 0.8$ & $14.3 / 1.4$ & $10.6 / 1.1$ & 5.0 thru 22.5/0.5 thru 2.2 \\
Meropenem/Netilmicin & $10 / 1$ & $15.3 / 1.5$ & $12.2 / 1.2$ & $21.4 / 2.1$ & $15.9 / 1.6$ & 7.9 thru 32.0/0.8 thru 3.1 \\
Meropenem/Amikacin & $6 / 1$ & $22.5 / 3.8$ & $18.8 / 3.1$ & $31.9 / 5.3$ & $23.8 / 4.0$ & 12.2 thru 46.4/2.0 thru 7.8 \\
\hline
\end{tabular}

Experiments were conducted three times using different platelets preparations. ${ }^{1}$ Ratio of concentrations of bioactive compounds in each mixture. ${ }^{2} \mathrm{C}_{50}$ values are expressed as $\mu \mathrm{g} / \mathrm{mL}$ of each antibiotic in the mixture that was added in the aggregometre cuvette. Final concentration of PAF in the aggregometer cuvette when tested in WRPs was $4.4 \times 10^{-11} \mathrm{M}$. WRPs: Washed Rabbit Platelets.

Both PAF and thrombin are implicated in severe inflammatory and coagulant procedures occurring during sepsis $[24,32]$. In addition it has been recently proposed that in chronic pathological states such as in cancers like melanoma, the PAF- and thrombin-activated pathways are interrelated, thus regulating, for instance, both the melanoma cell adhesion and its metastasis $[34,35]$. Critically ill patients often have systemic activation of both inflammation and coagulation [32]. Increasing evidence points to an extensive crosstalk between these two systems, whereby inflammation not only leads to activation of coagulation, but

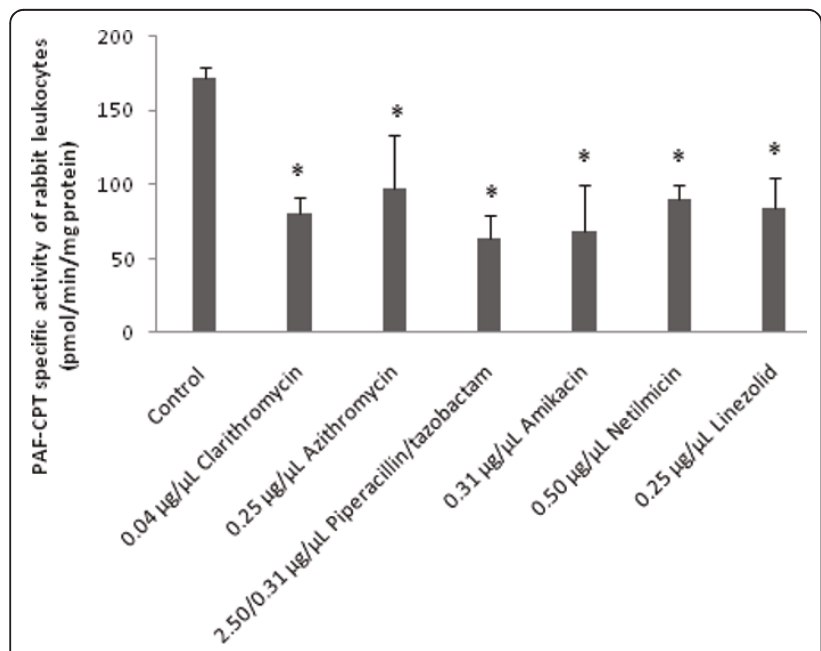

Figure 1 In vitro inhibitory effect of antibiotics towards PAFCPT enzyme activity of rabbit leukocytes. The amounts of each drug that induced approximately fifty to one hundred inhibitory effects against PAF-CPT specific activity are expressed as $\mu \mathrm{g}$ of each bioactive compound added in the assay mixture/ $\mu \mathrm{L}$ of assay volume. PAF-CPT specific activity of rabbit leukocytes is expressed as nmol of produced PAF/min/mg of total protein in assay. Control signifies PAF-CPT specific activity of rabbit leukocytes in the absence of any drug. Results are the average of three independent determinations using different enzyme preparations performing duplicate samples. ( ${ }^{*} p<0.05$ compared to control). PAF-CPT: Cholinephosphotransferase of PAF. coagulation also considerably affects inflammatory activity [32]. The intricate relationship between inflammation and coagulation may have major consequences for the pathogenesis of microvascular failure and subsequent multiple organ failure, as a result of severe infection and the associated systemic inflammatory response.

Beneficial effects have been observed as a result of treatment with various inhibitors or antagonists of PAF in different shock states and animal models [16-23]. To date, however, it has been impossible to translate these encouraging results from animal models in the clinical setting.

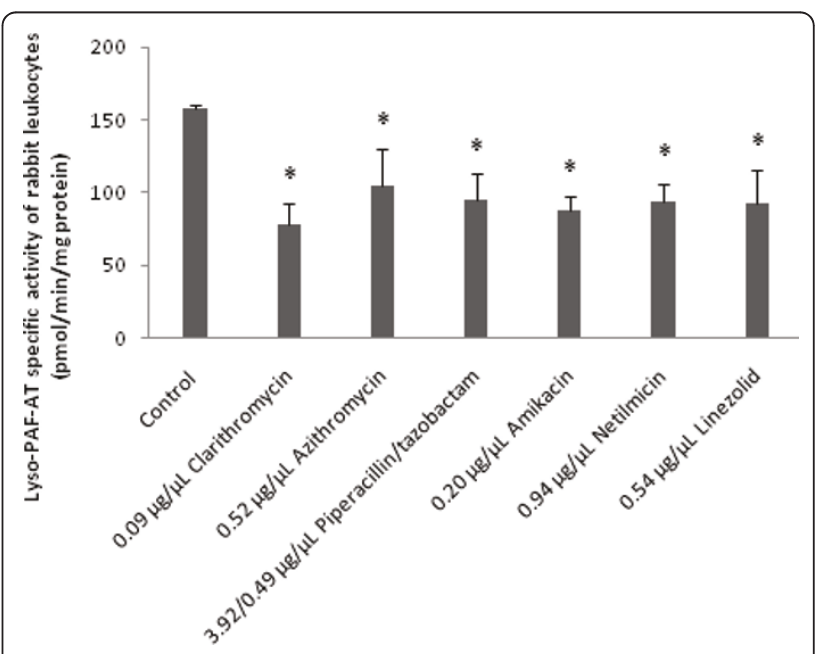

Figure 2 In vitro inhibitory effect of antibiotics towards LysoPAF-AT enzyme activity of rabbit leukocytes. The amounts of each drug that induced approximately fifty to one hundred inhibitory effects against Lyso-PAF-AT specific activity are expressed as $\mu \mathrm{g}$ of each bioactive compound added in the assay mixture/ $\mu \mathrm{L}$ of assay volume. Lyso-PAF-AT specific activity of rabbit leukocytes is expressed as nmol of produced PAF/min/mg of total protein in assay. Control signifies Lyso-PAF-AT specific activity of rabbit leukocytes in the absence of any drug. Results are the average of three independent determinations using different enzyme preparations performing duplicate samples. $\left({ }^{*} p<0.05\right.$ compared to control). 


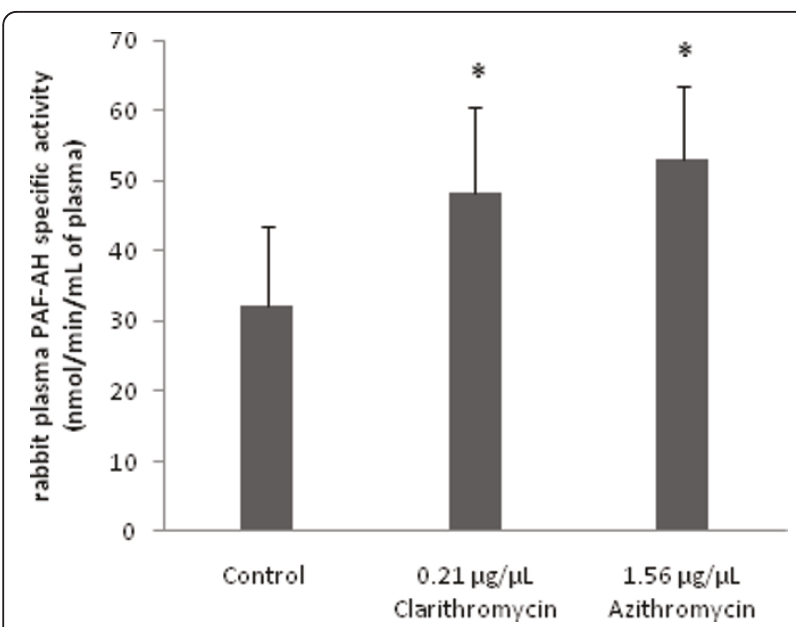

Figure 3 In vitro effect of antibiotics towards rabbit plasma PAF-AH enzyme activity. The amounts of each drug that induced significant increase on specific activity are expressed as $\mu \mathrm{g}$ of each bioactive compound added in the assay mixture/ $\mu \mathrm{L}$ of assay volume $(p<0.05$ versus control). Rabbit plasma PAF-AH specific activity is expressed as nmol of degraded PAF/min/mg of total protein in assay. Control signifies rabbit plasma PAF-AH specific activity of rabbit leukocytes in the absence of any drug. Results are the average of three independent determinations using different enzyme preparations performing duplicate samples. ${ }^{*} p<0.05$ compared to control). Plasma PAF-AH: plasma PAF-Acetylhydrolase

Recent studies in the field of gaining beneficial and promising results from an anti-PAF approach in several diseases have been focused in an effort not only to inhibit PAF action but also to down regulate its levels, through the inhibition of its biosynthesis and/or induction of its degradation $[14,15,25,26]$. For example, administration of rPAF-AH, protects mice from inflammatory injury and death after administration of lipopolysaccharide (LPS) or cecal ligation and puncture (CLP) [26]. Co-administration of antibiotics together with rPAF-AH was more protective than single treatment with either of these agents [26].

To our knowledge there are no other studies on the possible anti-inflammatory and anti-thrombotic properties of antibiotics used in sepsis treatment through their anti-PAF or anti-thrombin activities. This is the first study to report the anti-inflammatory and anti-thrombotic activities of a wide spectrum of antibiotics through their effects on PAF biological activities and its metabolism, as well as on thrombin. We also studied the effect of several of their combinations of treatment regimens in sepsis, against PAF activity.

In this study, in the case of the anti-PAF activities of the antibiotics tested, the biological assays were focused on the PAF-induced aggregation of both WRP's and rabbit PRP. In particular, our study on WRPs probes the anti-PAF activity of antibiotics under the experimental conditions applied, while, in the case of rabbit PRP, the conclusions drawn pinpoint the effect of these compounds on the PAF activation, similar to the in vivo conditions. In addition, the $\mathrm{IC}_{50}$ values measured in each case reflect the inhibition strength of each antibiotic, since a low $\mathrm{IC}_{50}$ value reveals stronger inhibition of the PAF-induced aggregation of either WRPs or rPRP for a given antibiotic concentration.

Our work leads to the conclusion that apart from their general anti-septic actions several antibiotics exhibit also a potent in vitro inhibitory effect against PAFinduced aggregation of both WRPs and rPRP, in a dosedependent manner (Tables 1 and 2). Significantly higher concentrations (at least one order of magnitude) of each compound were needed in order to inhibit the PAFinduced aggregation of rabbit PRP, compared to those needed in order to inhibit the corresponding aggregation of WRPs.

In the case of WRPs the antibiotics with the most prominent anti-PAF activity were clarithromycin, azithromycin, linezolid, amikacin and netilmicin, while in the case of rPRP were amikacin, azithromycin, tigecycline and clarithromycin. These results suggest that from all antibiotics tested in both WRPs and rPRP, the same three amikacin, azithromycin and clarithromycin, belonged to the ones with the most potent anti-PAF effect, even though higher concentrations of these drugs were needed in the case of rPRP. Only in the case of amikacin its $\mathrm{IC}_{50}$ values towards PAF-induced aggregation of both WRPs and rPRP were at the same order of magnitude.

Furthermore, tigecycline with one of the lowest antiPAF effects in WRPs exhibited a potent anti-PAF effect in the case of rPRP; only in this antibiotic its $\mathrm{IC}_{50}$ value towards PAF-induced aggregation of rPRP was approximately 5 times lower than that towards PAF-induced aggregation of WRPs. On the other hand, in the cases of linezolid and netilmicin with potent anti-PAF effects in WRPs, the first antibiotic did not inhibited PAF-induced aggregation of rPRP at all, while the second one exhibited one of the lowest anti-PAF effects in this case.

However, some of these drugs such as meropenem and vancomycin, did not influence PAF activity in WRPs, while the first one did not also inhibited PAFinduced aggregation of rPRP at all. Moreover, vancomycin induced in vitro aggregation of washed rabbit platelets, while cross-desensitization experiments showed that this platelet activation seems to take place through a different way than that of PAF-PAFR pathway.

It should also be noted that the anti-PAF activity of these drugs in WRPs was found similar to the most potent of other antimicrobial drugs that have been recently found to exhibit anti-PAF activity [15]. The $\mathrm{IC}_{50}$ values of these antibiotics against PAF share same 
or slightly less order of magnitude in comparison with the relatively $\mathrm{IC}_{50}$ values of some of the most potent PAF receptor-specific antagonists used in several models against sepsis and other diseases, such as WEB2170, BN52021, and rupatadine [18,36,37] $(0.009,0.013$, and $0.106 \mu \mathrm{g} / \mathrm{mL}$ in the aggregometer cuvette, respectively).

Moreover, some of these drugs seem to act synergistically against PAF-induced platelet aggregation in some but not in all combinations of treatment regimens against sepsis that were tested (Table 4). For example, when ceftazidime with the one of the lowest anti-PAF activity in WRPs $\left(\mathrm{IC}_{50}=28.79 \mu \mathrm{g} / \mathrm{mL}\right)$ was combined with either netilmicin with an $\mathrm{IC}_{50}$ value of $2.67 \mu \mathrm{g} / \mathrm{mL}$ or with amikacin with an $\mathrm{IC}_{50}$ value of $2.65 \mu \mathrm{g} / \mathrm{mL}$, the final mixture inhibited PAF-induced platelet aggregation with $\mathrm{IC}_{50}$ values of $10.6 / 1.1 \mu \mathrm{g} / \mathrm{mL}$ of the first or $9.9 / 1.7$ $\mu \mathrm{g} / \mathrm{mL}$ of the second mixture in the aggregometer cuvette respectively (Table 4 ). The synergistic anti-PAF action of these antibiotics when combined seems to belong to a more general pattern, since other antimicrobial drugs also when combined have been found to synergistically inhibit PAF [15]. It should be noted that the selection of antibiotic regimens tested was based on doses of these drugs that are usually administrated in patients, as well as from the $\mathrm{IC}_{50}$ values of each drug against PAF activity.

All antibiotics were additionally tested on the thrombin induced aggregation of WRPs. In the present study we have found also for the first time that several of these antibiotics exhibit additionally anti-thrombotic properties by inhibiting thrombin induced aggregation of WRPs in a concentration depended manner (Table 3 ). The antibiotics with the most prominent anti-thrombin activity were netilmicin and again azithromycin and amikacin. However, significantly higher concentrations (at least one order of magnitude, with the exception of netilmicin) of each compound were needed in order to inhibit the thrombin-induced aggregation of WRPs, compared to those needed in order to inhibit the corresponding PAF-induced aggregation of WRPs (Tables 1 and 2). This result points out that WRPs were actually viable and still normally functioning after incubation with concentrations of these antibiotics near their $\mathrm{IC}_{50}$ values towards PAF under the experimental conditions used, given that when platelets were incubated with much higher concentrations of these drugs they were aggregated normally when thrombin was used (in concentrations lower than their $\mathrm{IC}_{50}$ values towards thrombin).

In addition since much higher concentrations of these antibiotics were needed in order to $50 \%$ inhibit thrombin in WRPs, it seems that these drugs exhibit a more general anti-inflammatory action, which, however, is more specific towards the PAF-related pathway. Only in the case of netilmicin its $\mathrm{IC}_{50}$ value towards thrombin was in the same order of magnitude with that towards PAF; approximately 2 folds higher than that towards PAF. As a result this antibiotic exhibited the most potent inhibition towards thrombin, suggesting that netilmicin exhibits a more general anti-inflammatory and anti-thrombotic activity, since it can inhibit both the PAF and thrombin-related activities in concentrations in the same order of magnitude.

Taking into account all the above, one may suggest that apart from their general activities including their beneficial effects in sepsis, some of these drugs exhibit also a remarkable in vitro inhibitory effect against PAF or thrombin activities, while others did not affect PAF or thrombin activities, implying different perspectives for each antibiotic towards inflammatory and coagulant manifestations that usually occur during sepsis [21,32]. The observed differences between all drugs' inhibitory effects towards PAF and thrombin activities in different platelet preparations, WRPs and rPRP, point out dissimilar anti-inflammatory and/or anti-thrombotic potentials for each antibiotic and may be related to differences in their chemical structures and/or in their interactions with cell-membranes and/or plasma constituents.

Furthermore, in order to determine the possible interactions between these drugs and PAF metabolism, the in vitro effect of some of these drugs on the activities of PAF metabolic enzymes PAF-CPT, Lyso-PAF-AT and PAF-AH was also studied. For this purpose, we evaluated the specific activities of PAF-CPT and Lyso-PAF$\mathrm{AT}$ of homogenates of rabbit leukocytes, as well as rabbit plasma PAF-AH in the presence of each antibiotic in the assay mixture. We found for the first time that several of the antibiotics tested inhibited in vitro both PAF biosynthetic enzymes in a concentration depended manner (Figures 1 and 2), while only clarithromycin and azithromycin induced an in vitro increase of rabbit plasma PAF-AH, in concentrations an order of magnitude higher than those of PAF-biosynthesis inhibition and their $\mathrm{IC}_{50}$ values against PAF (Figure 3).

Smaller amounts (one to two order of magnitude) of clarithromycin were needed in order to fifty to one hundred inhibit PAF-CPT and Lyso-PAF-AT specific activities, in relevance to the other drugs tested. This result, aided by the facts that this antibiotic seems to induce PAF-degradation in lower concentrations than the other antibiotics tested and potently inhibit PAF-induced platelet aggregation, propose a promising role for this drug as far as concerns its potent anti-inflammatory activity in sepsis.

Moreover, the amounts of all antibiotics that were needed in order to fifty to one hundred inhibit LysoPAF-AT specific activity were twice higher than those for the relevant inhibition of PAF-CPT, except for 
amikacin, where lesser amounts were needed. This result may be a sign of irreconcilable differences in the inhibitory effect of these antibiotics against the two distinct biosynthetic routes of PAF. Taking also into account that amikacin exhibited one of the most potent anti-PAF effects (this antibiotic was the only one that its low $\mathrm{IC}_{50}$ values were in the same order of magnitude towards PAF-induced aggregations of both WRPs and rPRP) and one of the most potent anti-thrombotic effects, the additional potent inhibitory effect of this antibiotic towards PAF-biosynthesis provide new antiinflammatory potentials for this drug.

Taking into account that during sepsis PAF synthesis is induced by bacteria LPS through toll-like receptors [33], the inhibitory effect of some of these drugs against PAF biosynthetic enzymes may reduce PAF-synthesis, down regulating thus PAF-activity and subsequently PAF-related inflammatory procedures.

\section{Conclusions}

This is the first study to bring in surface putative antiinflammatory and anti-thrombotic activities of some antibiotics used in sepsis, through their in vitro studied anti-PAF and anti-thrombin effects in rabbit platelets. Furthermore, these drugs have exhibited the ability to inhibit also PAF-synthesis. Amicacin, clarithromycin and azithromycin with the most potent anti-PAF activities in both WRPs and rPRP, showed the most potent inhibitory effect also towards PAF-biosynthesis, while clarithromycin and azithromycin were the only ones that could induce PAF-degradation. Amikacin also inhibited potently thrombin.

It seems that these newly found anti-inflammatory and anti-thrombotic properties of antibiotics and/or antibiotic regimens used in sepsis, such as their inhibitory activities towards PAF/PAFR and thrombin pathways, as well as their interactions with PAF-metabolism, may provide new perspectives for these drugs towards also the inflammatory and coagulant manifestations that usually take place during several septic stages, including induced by severe sepsis multiple organ failure.

However, more in vitro and in vivo tests in animal models are needed in order to confirm which of the antibiotic regimens used in sepsis may exhibit the most potent anti-inflammatory effect through the highest in vivo inhibitory effect against PAF activities and biosynthesis, with simultaneously induction of PAF-degradation, in an effort to increase our understanding of the clinical implications of PAF inhibition with regard to septic shock, severe sepsis and induced multiple organ failure. In another point of view, the simultaneous coadministration of antibiotic regimens with specific PAF antagonists/drugs and/or recombinant PAF-AH should also be considered and may augment the efficacy of antibiotic treatment of sepsis.

The present study is the first step in this direction, while combined with the outcomes of the future in vivo studies it may optimize the efficacy of antibiotic treatment in inflammatory septic conditions.

\section{Acknowledgements}

This work was partially supported by grants from the Greek State Scholarships Foundation (A.B. Tsoupras is a holder of a postdoctoral scholarship in the field of biochemistry from this institution) and from the Hellenic Society for the research, study, and education in infectious diseases.

\section{Author details}

${ }^{1}$ Faculty of Chemistry, National \& Kapodistrian University of Athens, Panepistimioupolis of Zografou, Athens, 15771, Greece. ${ }^{2} 3$ rd Internal Medicine Dept.-Infectious Diseases Unit, Red Cross General Hospital, Athens, Greece.

\section{Authors' contributions}

ABT conceived of the study, participated in its design and coordination, carried out the in vitro studies including the biological test in rabbit platelets, the separation of cells and plasma from rabbit blood, PAFmetabolic enzymes tests, and drafted the manuscript. MC participated in the design of the study. AL participated in the design of the study. GT participated in the design of the study. NT participated in the design of the study and helped to draft the manuscript. CAD conceived of the study, participated in its design and coordination and helped to draft the manuscript. MCL conceived of the study and participated in its design and coordination. All authors have read and approved the final manuscript.

\section{Competing interests}

The authors declare that they have no competing interests.

Received: 23 August 2010 Accepted: 7 July 2011 Published: 7 July 2011

\section{References}

1. Demopoulos CA, Pinckard RN, Hanahan DJ: Platelet-activating factor. Evidence for 1-0-alkyl-2-acetyl-sn-glyceryl-3-phosphorylcholine as the active component (a new class of lipid chemical mediators). Journal of Biological Chemistry 1979, 254:9355-9358.

2. Zimmerman GA, Mclntyre TM, Prescott SM, Stafforini DM: The plateletactivating factor signaling system and its regulators in syndromes of inflammation and thrombosis. Crit Care Med 2002, 30(Suppl 5):294-301.

3. Stafforini DM, Mclntyre TM, Zimmerman GA, Prescott SM: Plateletactivating factor, a pleiotrophic mediator of physiological and pathological processes. Crit Rev Cl Lab Sci 2003, 40:643-672.

4. Snyder F: Platelet-activating factor and its analogs: metabolic pathways and related intracellular processes. Biochim Biophys Acta 1995, 1254:231-249.

5. Francescangeli E, Boila A, Goracci G: Properties and regulation of microsomal PAF-synthesizing enzymes in rat brain cortex. Neurochem Res 2000, 25:705-713.

6. Snyder F: CDP-choline:alkylacetylglycerol cholinephosphotransferase catalyzes the final step in the de novo synthesis of platelet-activating factor. Biochim Biophys Acta 1997, 1348:111-116.

7. Stafforini DM, McIntyre TM, Carter ME, Prescott SM: Human plasma platelet-activating factor acetylhydrolase. Association with lipoprotein particles and role in the degradation of platelet-activating factor. J Biol Chem 1987, 262:4215-4222.

8. Montrucchio G, Alloatti G, Camussi G: Role of platelet-activating factor in cardiovascular pathophysiology. Physiol Rev 2000, 80:1669-1699.

9. Demopoulos CA, Karantonis HC, Antonopoulou S: Platelet activating factor-a molecular link between atherosclerosis theories. European Journal Of Lipid Science And Technology 2003, 105:705-716.

10. López-Novoa JM: Potential role of platelet activating factor in acute renal failure. Kidney Int 1999, 55:1672-1682. 
11. McManus LM, Pinckard RN: PAF, a putative mediator of oral inflammation. Crit Rev Oral Biol Med 2000, 11:240-258.

12. Kasperska-Zajac A, Brzoza Z, Rogala B: Platelet-activating factor (PAF): a review of its role in asthma and clinical efficacy of PAF antagonists in the disease therapy. Recent Pat Inflamm Allergy Drug Discov 2008, 2:72-76.

13. Nathan N, Denizot Y, Huc MC, Claverie C, Laubie B, Benveniste J, Arnoux B Elevated levels of paf-acether in blood of patients with type 1 diabetes mellitus. Diabete Metab 1992, 18:59-62.

14. Tsoupras AB, latrou C, Frangia C, Demopoulos CA: The implication of platelet activating factor in cancer growth and metastasis: potent beneficial role of PAF-inhibitors and antioxidants. Infect Disord Drug Targets 2009, 9:390-399

15. Tsoupras AB, Chini M, Tsogas N, Fragopoulou E, Nomikos T, Lioni A, Mangafas N, Demopoulos CA, Antonopoulou S, Lazanas MC: Anti-plateletactivating factor effects of highly active antiretroviral therapy (HAART): a new insight in the drug therapy of HIV infection? AIDS Res Hum Retroviruses 2008, 24:1079-1086.

16. Fink MP: Therapeutic options directed against platelet activating factor, eicosanoids and bradykinin in sepsis. J Antimicrob Chemother 1998, 41(Suppl A):81-94

17. Moreno SE, Alves-Filho JC, Rios-Santos F, Silva JS, Ferreira SH, Cunha FQ, Teixeira MM: Signaling via platelet-activating factor receptors accounts for the impairment of neutrophil migration in polymicrobial sepsis. $J$ Immunol 2006, 177:1264-1271.

18. Heuer HO: Involvement of platelet-activating factor (PAF) in septic shock and priming as indicated by the effect of hetrazepinoic PAF antagonists. Lipids 1991, 26:1369-1373.

19. Tetta C, Mariano F, Buades J, Ronco C, Wratten ML, Camussi G: Relevance of platelet-activating factor in inflammation and sepsis: mechanisms and kinetics of removal in extracorporeal treatments. Am J Kidney Dis 1997, 30(5 Suppl 4):57-65.

20. Graham RM, Strahan ME, Norman KW, Watkins DN, Sturm MJ, Taylor RR: Platelet and plasma platelet-activating factor in sepsis and myocardial infarction. J Lipid Mediat Cell Signal 1994, 9:167-182.

21. Ayala A, Chaudry $\mathrm{IH}$ : Platelet activating factor and its role in trauma, shock, and sepsis. New Horiz 1996, 4:265-275.

22. Anderson $\mathrm{BO}$, Bensard $\mathrm{DD}$, Harken $\mathrm{AH}$ : The role of platelet activating factor and its antagonists in shock, sepsis and multiple organ failure. Surg Gynecol Obstet 1991, 172:415-424.

23. Suputtamongkol $Y$, Intaranongpai $S$, Smith MD, Angus $B$, Chaowagul $W$, Permpikul C, Simpson JA, Leelarasamee A, Curtis L, White NJ: A doubleblind placebo-controlled study of an infusion of lexipafant (Plateletactivating factor receptor antagonist) in patients with severe sepsis. Antimicrob Agents Chemother 2000, 44:693-696.

24. Negro Alvarez JM, Miralles López JC, Ortiz Martínez JL, Abellán Alemán A, Rubio del Barrio R: Platelet-activating factor antagonists. Allergol Immunopathol 1997, 25:249-258.

25. Tsoupras AB, Fragopoulou E, Nomikos T, latrou C, Antonopoulou S, Demopoulos CA: Characterization of the de novo biosynthetic enzyme of platelet activating factor, DDT-insensitive cholinephosphotransferase, of human mesangial cells. Mediators Inflamm 2007, 2007:27683.

26. Gomes RN, Bozza FA, Amâncio RT, Japiassú AM, Vianna RC, Larangeira AP, Gouvêa JM, Bastos MS, Zimmerman GA, Stafforini DM, Prescott SM, Bozza PT, Castro-Faria-Neto HC: Exogenous platelet-activating factor acetylhydrolase reduces mortality in mice with systemic inflammatory response syndrome and sepsis. Shock 2006, 26:41-49.

27. Demopoulos CA, Koussissis S, Lazanas M, Lakrakis-Lazanas K: PAF of biological fluids in disease: I. Levels in blood and urine in cancer. Clin Chem Enzymol Commun 1990, 3:41.

28. Tsoukatos D, Demopoulos CA, Tselepis AD, Moschidis MC, Donos A, Evangelou A, Benveniste J: Inhibition of cardiolipins of platelet-activating factor-induced rabbit platelet activation. Lipids 1993, 28:1119-1124.

29. Bligh EG, Dyer WJ: A rapid method of total lipid extraction and purification. Can J Biochem Physiol 1959, 37:911-917.

30. Antonopoulou S, Demopoulos CA, latrou C, Moustakas G, Zirogiannis P: Platelet-activating factor acetylhydrolase (PAF-AH) in human kidney. Int $J$ Biochem 1994, 26:1157-1162

31. Bradford MM: A rapid and sensitive method for the quantization of microgram quantities of protein utilizing the principle of protein-dye binding. Anal Biochem 1976, 72:248-254
32. Levi $\mathrm{M}$ : The coagulant response in sepsis and inflammation. Hamostaseologie 2010, 30:10-12, 14-16.

33. Shimizu T: Lipid mediators in health and disease: enzymes and receptors as therapeutic targets for the regulation of immunity and inflammation. Annu Rev Pharmacol Toxicol 2009, 49:123-150.

34. Melnikova VO, Balasubramanian K, Villares GJ, Dobroff AS, Zigler M, Wang H, Petersson F, Price JE, Schroit A, Prieto VG, Hung MC, Bar-Eli M: Crosstalk between protease-activated receptor 1 and platelet activating factor receptor regulates melanoma cell adhesion molecule (MCAM/MUC18) expression andmelanomametastasis. Journal of Biological Chemistry 2009, 284:28845-28855.

35. Melnikova VO, Bar-Eli M: Inflammation and melanoma metastasis. Pigment Cell and Melanoma Research 2009, 22:257-267.

36. Fletcher JR, Disimone AG, Earnest MA: Platelet activating factor receptor antagonist improves survival and attenuates eicosanoid release in severe endotoxemia. Ann Surg 1990, 211:312-316.

37. Izquierdo I, Merlos M, García-Rafanell J: Rupatadine: a new selective histamine $\mathrm{H} 1$ receptor and platelet-activating factor (PAF) antagonist. A review of pharmacological profile and clinical management of allergic rhinitis. Drugs Today 2003, 39:451-468.

doi:10.1186/1476-9255-8-17

Cite this article as: Tsoupras et al.: In vitro anti-inflammatory and anticoagulant effects of antibiotics towards Platelet Activating Factor and thrombin. Journal of Inflammation 2011 8:17.

\section{Submit your next manuscript to BioMed Central and take full advantage of:}

- Convenient online submission

- Thorough peer review

- No space constraints or color figure charges

- Immediate publication on acceptance

- Inclusion in PubMed, CAS, Scopus and Google Scholar

- Research which is freely available for redistribution

Submit your manuscript at www.biomedcentral.com/submit
Ciomed Central 